Editorial

\title{
A brief discussion of association of radiation and metabolism
}

Volume 5 Issue 3 - 2016

Xin Wang
ACURE Biotechnology, INC., USA

\section{Editorial}

Since Marie Curie isolated relatively pure radioactive materials from magnetic rocks, it has been longer than a century that radiation is applied and now full of our life. We expose under radiation from not only the sun and radiation medicine, but also from information receiving equipments like computers, phones where waves could be enhanced in radioactive 'CHANG'--same as electric field or human circumstances. The unpleasant effects from radioactivity had been a top topic to study in many fields of science. Here, I briefly introduce my opinion on the association of radioactivity and metabolism through summarizing the research results of radiology. I believe that the metabolism, either the effects of radiation on metabolism of living body or the metabolism of radiation from living beings are the keys making us get along with radioactivity.

Diversity of effects of radiation on distinct cell types suggest that mitochondria could be the most important organelle in metabolism of radiation. Mitochondria is known as metabolic machine of cells providing energy. According to the amount of mitochondria content, cells work with different functions. For example, skin cells present fewer amount of mitochondria than heart muscle cells. Inner (basal) layer of skin cells reproduce self and grow to outer layers slowly. Outer layers of skin cells' mitochondria disappear, gradually die and keratinize to protect inner tissue. Light, sometimes only sun radiation exposure of skin cells can cause inflammation, keratinized layers peel off, basal reproductive skin cells over grow, even genetic mutate to form skin tumor. However, not higher risk rate of skin tumor in children, it maybe because the earlier and severer inflammation alert and avoid the young people expose in long high dose of radioactivity.

Heart muscle cells contain a lot of mitochondria to produce ATP for restless heart beating. The producing process of energy in mitochondria actually is a progress of transfer and deliver the charges through the middle products of metabolism in both catabolic and anabolic processes. Radiation are extra charged particles run away from the matter to reach a balance between positive and negative charges, in Chinese they like to describe it as 'YIN YANG'. Let us image that when the charged particles run into the charged middle metabolic products in mitochondria, they may have the potential to transport their own charges and be involved in metabolism of heart muscle cells. This may explain why moderated radiation exposure seems keep this organ younger and working longer, and reduced cardiovascular disease--let us look at what seems be happening in Japan. Of course, we need to consider the Japanese diet at same time. This is very interesting and we will develop radiational devices for prevention and treatment of cardiovascular diseases.
Correspondence: Xin Wang, ACURE Biotechnology, INC., Ellicott City, Maryland, USA, Email acurebiotech@gmail.com

Received: March 12, 2016 | Published: March 28, 2016
For neurons, the most reported side effects of radiation are depression and neuron degeneration and tumor. Mitochondria in brain use only glucose as energy provider. Because the protection of skull and blood-brain battery (BBB), very few radiation have capacity to penetrate to reach the neurons. However, the damage is significant when neurons got exposed under radiation. Bone is a organ formed by bone cells. Similar with skin cells, bone cells have mitochondria containing reproductive bone cell layers at two terminal of long bones. Mitochondria disappear, bone cell die and calcificate with calcium in the middle long bones. Calcium harden the bone, protect the inner hematopoietic bone marrow. For young children, their bones are still in a fast growing phase, the reproductive bone cell lays are more active than adult, and calcification is not enough, radiation can cause bone tumor and leukemia to young population. Because the skull of growing children are not mature, radiation bring children in high risk of the brain tumor.

\section{Conclusion}

In summary, the harmfulness of radiation is closely related to metabolism. As the economic power source, if radiation will be applied for more population, we need to find easy handle approaches to prevent vulnerable people from radiation exposure first, because leaking of radiation causes deadly diseases, especially it is dangerous for our next generation, the future of the Earth.

\section{Acknowledgments}

None.

\section{Conflicts of interest}

Author declares that there is no conflict of interest. 